\title{
Oral Doxycycline on the Level of Matrix Metalloproteinase 9 in Rat Models Experiencing Traumatic Brain Injury
}

\author{
Daud K.R. ${ }^{1}$, Islam A.A. ${ }^{2}$, Ihwan A. ${ }^{2}$, Alfian Zainuddin A.A. ${ }^{3}$, Faruk M. ${ }^{1}$ \\ ${ }^{1}$ Department of Surgery, Faculty of Medicine, Hasanuddin University, Jl., Makassar, Indonesia. \\ ${ }^{2}$ Division of Neurosurgery, Department of Surgery, Faculty of Medicine, Hasanuddin University, Makassar, Indonesia \\ ${ }^{3}$ Department of Public Health and Community Medicine Science, Faculty of Medicine, Hasanuddin University, Makassar, Indonesia
}

\begin{abstract}
INTRODUCTION: Cerebral edema in traumatic brain injury (TBI) results from hyperpermeability of the bloodbrain barrier (BBB). One of the causes in BBB permeability disorders is the activation of matrix metalloproteinase 9 (MMP-9) which can be inhibited by the administration of doxycycline. Knowing the role of oral doxycycline administration as an inhibitor of MMP-9 activation on the level of MMP-9 in cerebral edema in traumatic brain injury. MATERIALS AND METHODS: This study was an experimental study in Wistar rats with a post-test control design consisting of one control group, one group with provoked brain injury, and one group with provoked brain injury followed by oral doxycycline administration. RESULT: There were significant differences in the level of MMP -9 between the control group, the provoked brain injury group and the provoked brain injury group followed by oral doxycycline administration with $\mathrm{p}$-value $=0.01(\mathrm{p}<0.05)$. CONCLUSION: Oral doxycycline administration can inhibit the increase of MMP-9 levels in cerebral edema in traumatic brain injury in Wistar rats.
\end{abstract}

KEYWORDS: MMP-9, Doxycycline, Traumatic Brain Injury, Marmarou Model.

\section{INTRODUCTION}

Traumatic brain injury is a complex disorder as a result of a change in brain function or brain pathology caused by external force. ${ }^{1}$ The major complication of a traumatic brain injury is cerebral edema. ${ }^{2}$ It is a pathological state with increased brain volume due to abnormally accumulated fluid in cerebral parenchym. ${ }^{3}$ Vasogenic edema is an increase in extra-cellular fluids due to the leakage of blood brain barrier (BBB) resulting in an increase in osmotic pressure and causing fluid to flow out of the blood vessels and into the extra-cellular compartments. ${ }^{4}$

Matrix Metalloproteinase (MMP) is a zinc-dependent endopeptidase enzyme that plays a role in tissue repair. MMP regulation is very complex and controlled. The

Corresponding Author:

Dr. Muhammad Faruk

Department of Surgery,

Faculty of Medicine, Hasanuddin University,

Jl. Perintis Kemerdekaan KM 11, Makassar,

90245, Indonesia.

Tel No : +62411-585984

E-mail : faroex8283@gmail.com lack of this regulatory control will play a role in the pathophysiology of BBB damage in cerebral edema. Hence, the MMP-9 inhibition becomes an important target in the treatment of traumatic brain injury. ${ }^{2}$

Currently, research on tetracycline discovered its essential pharmacological properties that focus on inflammation pathway. The broad spectrum of the antiinflammatory effect of tetracycline is caused by its ability to inhibit synthesis or activities of some inflammatory mediators ${ }^{5}$, such as MMPs, prostaglandin (PGE), and cytokine (tumor necrosis factor alpha, interleukin-(IL-) 1, IL6). ${ }^{6}$ Doxycycline is the most powerful non-specific MMP inhibitor in the tetracycline derivative that can inhibit MMP-9.7,8 Based on these facts, this study attempts to reveal the extent of the role of oral doxycycline on levels of MMP in traumatic brain injury.

\section{MATERIALS AND METHODS}

This study was an experimental laboratory model on rats with post-test control group design that aimed to find

IMJM Volume 20 No.1, Jan 2021 
out the role of oral doxycycline to levels of MMP-9 in cerebral edema after a traumatic brain injury in rats. All animal procedures received approval from Research Ethics Committee, Faculty of Medicine, University of Hasanuddin, Makassar, Indonesia, under Number: 1113/UN4.6.4.5.31/PP36/2019.

Doxycycline was purchased from Dexa-Medica Pte.ltd, and Rat MMP-9 (ELISA) was obtained from Bioassay Technology Laboratory, Shanghai Korain Biotech Co., Ltd, Shanghai, China with catalog No. E0321Ra.

The study was done in an animal laboratory of Medical Faculty at University of Hasanuddin within two months. The sample populations were divided into three groups which are: group 1 as the control group without provoked brain injury, group 2 as a group with provoked brain injury where blood samples was collected 24 hours afterwards, and group 3 as the group with provoked brain injury which was given a dosage of $30 \mathrm{mg} / \mathrm{kg}$ oral doxycycline afterwards which wasthen followed by the collection of 27 hours blood samples 27 -hours later.

The samples for each group was 9 experimental rats with the inclusion criteria of male Wistar rats (Rattus norvegicus), approximately 3-4 months old, weight 300400 grams, and in healthy condition. The exclusion criteria were animals that appeared unhealthy during adaptation period, losing weight $>10 \%$ during the adaptation. The drop out criteria were dying before the study was finished and damaged blood samples.

\section{Surgical Procedure}

Samples were inflictd with brain injury in accordance to the modified Marmarou model,, 10 where the animals were anesthetized with $3 \%$ sodium pentobarbital (50 $\mathrm{mg} / \mathrm{kg}$ ), and then placed in a stereotaxic frame. Midline incision was performed, the skin and temporal muscles are reflected to open the skull. Craniotomy was performed using a dental burr with a diameter of 4.0 $\mathrm{mm}$, A controlled cortical impact model tool is used to put pressure on the brain (tip diameter, $4.0 \mathrm{~mm}$; depth of cortical contusion, $5.0 \mathrm{~mm}$; impact speed, $6.0 \mathrm{~m} / \mathrm{s}$; residence time, $50.0 \mathrm{~ms}$ ). After the injury, the skin incision is closed with nylon sutures.
At the end of the procedure, the animals are removed from the stereotaxic frame, returned to their cages and monitored closely until their recovery from anesthesia was complete.

\section{Doxycycline Administration}

The rats from group 3 were administered with $30 \mathrm{mg} / \mathrm{kg}$ body weight of oral doxycycline. This dose has been shown previously to suppress cerebral MMP-9 activity in rodents and to reduce ischemic brain damage.

\section{Sample Collection and Examination}

The blood is centrifuged and the resulting supernatant, designated plasmawas immediately transfered into a clean polypropylene tube using a Pasteur pipette. The samples were then maintained at $2-8^{\circ} \mathrm{C}$.

The MMP-9 levels were measured by ELISA method classified into a span of a certain concentration which were $0.01 \mathrm{ng} / \mathrm{ml}, 0.08 \mathrm{ng} / \mathrm{ml}, 0.2 \mathrm{ng} / \mathrm{ml}, 0.4 \mathrm{ng} / \mathrm{ml}$ and $0.8 \mathrm{ng} / \mathrm{ml}$. Levels of MMP-9 would then be compared between each sample group.

\section{Statistical Analysis}

All data were processed and analyzed using SPSS version 22 (IBM Corp. Released 2013. IBM SPSS Statistics for Windows, Version 22.0. Armonk, NY: IBM Corp.). Normally distributed variables were expressed as means \pm standard deviations unless indicated otherwise and one-way variant analysis ANOVA as well as post hoc test with LSD method for parametric data.

\section{RESULTS}

The sample consisted of 3 groups with 9 rats each for the control group, the traumatic brain injury group without oral doxycycline given (TBI), and the group of traumatic brain injury with oral doxycycline administered (TBI+D). Twenty-seven rats had met inclusion criteria, not one had the exclusion criteria. All groups have data of MMP-9 levels. Data collected are numerical data that are distributed normally, which were tested by ANOVA test. 
Table 1 shows that matrix metalloproteinase levels on the TBI group has a mean of 0.5939 with standard deviation of $0.05944 \mathrm{ng} / \mathrm{ml}$ compared with control group that has a mean of $0.5219 \mathrm{ng} / \mathrm{ml}$ with standard deviation of $0.4895 / \mathrm{ml}$. This can also be seen from the Boxplot graph below (Figure 1).

Table 1. Matrix metalloproteinase 9 levels by group

\begin{tabular}{lccccl}
\hline \multicolumn{6}{l}{ Matrix metalloproteinase 9 levels $(\mathrm{ng} / \mathrm{ml})$} \\
\hline Group & $\mathrm{N}$ & Mean (SD) & Median & Min-Max & $p$ \\
Control & 9 & 0.5219 & 0.5175 & $0.4271-$ & \\
& & $(0.04895)$ & & 0.6058 & \\
& & & & & 0.01 \\
TBI & 9 & $\begin{array}{l}0.5939 \\
\text { (0.05944) }\end{array}$ & 0.6046 & $0.5045-$ & 4 \\
& & & 0.6813 & \\
TBI+D & 9 & 0.5371 & 0.5295 & $0.4582-$ & \\
& & $(0.04013)$ & & 0.6047 & \\
\hline
\end{tabular}

ANOVA test found that the result was significant with $\mathrm{p}$-value $=0.014(\mathrm{p}<0.05)$. There was a statistically significant relation in administering oral doxycycline to a reduction of MMP-9 level in cerebral edema on Wistar rats after a traumatic brain injury with $\mathrm{p}<0.05$.

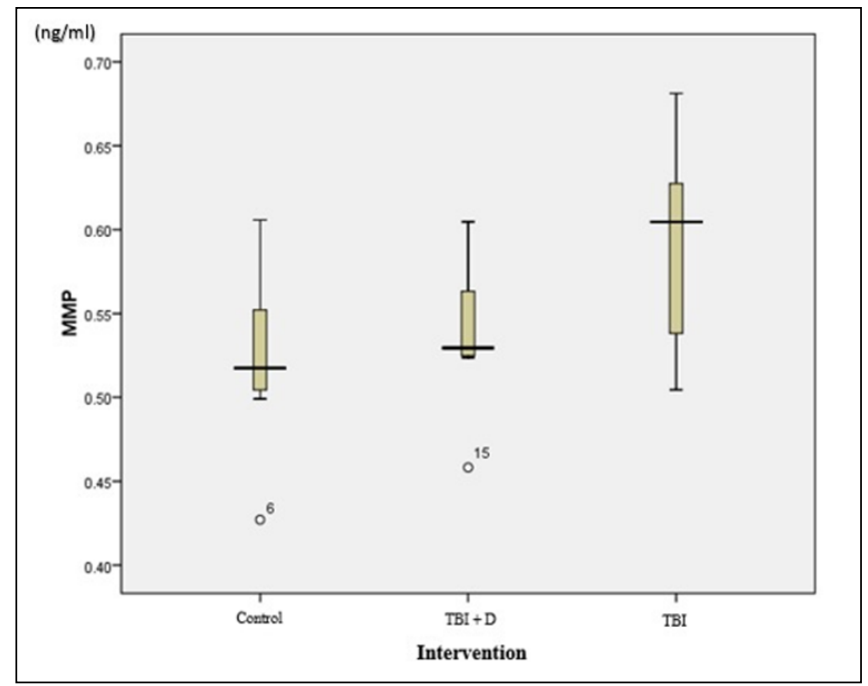

Figure 1. MMP-9 serum levels in rats with and without doxycycline administered.

Table 2 shows that the increase of MMP-9 levels on the TBI group was statistically significant with $\mathrm{p}$ value $=$ 0.006. Meanwhile, there was a difference of MMP-9 level on TBI+D group to the TBI group which was significant with $\mathrm{p}=0.02$. Table 2 was the result of the second data test using LSD method.

Table 2. Post hoc test with LSD method

\begin{tabular}{lll}
\hline Group & Group & $\mathrm{P}$ \\
& & $<0,05$ \\
\hline \multirow{2}{*}{ Control } & TBI+D & 0.052 \\
& TBI & 0.006 \\
TBI+D & TBI & 0.022 \\
\hline
\end{tabular}

\section{DISCUSSION}

The total number of samples in each group in this study was 9 Wistar rats, which was based on the Formula of Federer. Traumatic brain injury was performed on two experimental groups, which are TBI and TBI+D using Feeney's drop model (weight drop traumatic brain injury model) to obtain the effect of blood brain barrier damage induced by a traumatic brain injury on the animals. $^{11}$

The results indicate that 24 hours after provoked brain injury using 571.17 Newton $/ \mathrm{mm} 2$ in the area of craniectomy on group TBI shows significant elevation of MMP-9 level $(p<0.05)$ against the control group. This is considered in accordance with study of Hayashi et al in 2009 which showed MMP-9 level increased significantly $(\mathrm{p}<0.05)$ in 24 and 48 hours after traumatic brain injury. ${ }^{12}$

Other study also show that although it is classified in the acute phase, MMP-9 increment is not significant $(p>0.05) 1$ hour after a traumatic brain injury. ${ }^{12}$ The level of MMP-9 will rise as a result of ischemic reperfusion in secondary injury. Secondary injuries occur within minutes of a traumatic brain injury, and can hold out for months or years and play a major role in the morbidity and mortality resulting from traumatic brain injury. ${ }^{13,14}$ To know the actual period of MMP level increment in the serum is essential, since the right time to intervene in impeding MMP is when MMP levels are destabilized after the traumatic brain injury. ${ }^{12}$ This then became the basis of MMP-9 levels not being measured 1 hour after the provoked brain injury in this study.

An increase in MMP level will cause blood brain barrier hyperpermeability which causes cerebral edema. ${ }^{15}$ To prevent tissue damage, MMP activity must then be tightly controlled. Treatment strategies for traumatic brain injury includes inhibition of matrix metalloproteinases with hypothermia and hyperbaric oxygen therapy with satisfactory results, ${ }^{12}$ administration of rhubarb (rhizome) drug; which is a type of traditional medicine, ${ }^{16}$ administration of minocycline which can inhibit MMP by completely blocking the damaging effects, and administration of intravenous doxycycline which has also been shown to reduce MMP levels. ${ }^{17}$

IMJM Volume 20 No.1, Jan 2021 
Our data showed that doxycycline treatment was able to reduce MMP-9 at 24 hours after TBI. The BBB opening in immature brain plays a vital role in the severity of brain edema. As endothelial cells and the end-foot of astrocytes are the major structural components of $\mathrm{BBB}$, to confirm the cell-types and sources of MMP-9 expression, blood sampling was performed at $24 \mathrm{~h}$ after injury. ${ }^{18}$

Doxycycline is a broad-spectrum tetracycline antibiotic widely used in the clinic. Besides its antibiotic function, doxycycline has well-recognized anti-MMP properties which are not well defined but appeared to be through inhibition of both MMP synthesis and activity (e.g., MMP-2). Doxycycline was used as a broad inhibitor of MMPs to effectively inhibit multiple MMPs. ${ }^{19}$

Tetracycline inhibits the process of transcription and activation of MMP. ${ }^{20}$ This process takes place probably due to its ability in binding with zinc from some active part of MMP, as MMP needs zinc itself to be activated. ${ }^{21}$

Tetracycline antibiotics such as doxycycline have important anti-inflammatory activities, and this could potentially reduce the damage after ischemia. ${ }^{22}$ Even though doxycycline was not present in the animals at the time of ischemia, it is possible that the antiinflammatory effects are longer lasting. Another important effect is protection of the blood-brain-barrier (BBB). MMPs have been implicated in BBB disruption following transient ischemia and hemorrhagic transformation after reperfusion. ${ }^{23}$

In this study there was a significant difference in $\mathrm{TBI}+\mathrm{D}$ when compared to the group TBI $(\mathrm{p}=0.022)$. This is probably due to the reduced level of apoptosis and oxidative stress in the distinct anatomical areas examined and the resulting diminished infiltration of immune cells in parenchymal areas. As an established MMP blocker, doxycycline reduced the degradation of junctional complex proteins in parenchymal vessels. In addition, doxycycline treatment was associated with an overall reduction in the expression and activity of MMPs, particularly in areas of leukocyte infiltration. These results indicate that a broad-range inhibitor of MMPs promotes host survival and suggest the potential of doxycycline as a therapeutic agent for the control of inflammatory responses associated with neurocysticercosis. ${ }^{24}$ Doxycycline also reduced MMP activity, as gelatin and collagen degradation was lower in leukocytes infiltrating leptomeninges. ${ }^{24}$

Other studies show that ischemic animals treated with doxycycline exhibited a significant reduction in the expression of the plasminogen activator uPA, and this was associated with decreased damage of the BBB. ${ }^{25}$ Likewise, in an infant rat model of pneumococcal meningitis, doxycycline significantly inhibited the production of pro-inflammatory mediators, which reduced $\mathrm{BBB}$ disruption and the extent of cortical brain injury. ${ }^{26}$

Doxycycline could be directly inhibiting MMPs by nonmutually exclusive mechanisms such as preventing the in-vivo activation of pro-MMP zymogens by the MT1MMP, blocking the active site or inducing conformational changes by binding to the $\mathrm{Zn} 2+$ or $\mathrm{Ca} 2+$ sites, dampening the inflammatory response associated with MMP up-regulation, or preventing MMP activation by reducing levels of plasminogen activator. ${ }^{24}$

Damian $\mathrm{N}$ et al used a dose of 30 milligrams per kilogram of weight for their research in reducing mortality and rates of brain and cochlear injury in pneumococcal meningitis in 2006.21, 27 In this study, doxycycline are administered with the dosage of 30 milligrams per kilogram body weight orally on group $\mathrm{TBI}+\mathrm{D}$ to see its role on MMP-9 levels.

The study shows that the difference of MMP-9 level among the TBI+D group, TBI group and control group are significant with $\mathrm{p}$ value $=0.01(\mathrm{p}<0.05)$. Once tested with the post hoc test with LSD method, the $\mathrm{TBI}+\mathrm{D}$ group has a value of $\mathrm{p}=0.022$ when compared to the TBI group. It is suggested that MMP-9 levels are declining in a post-traumatic brain injury after administering oral doxycycline. Doxycycline has faster oral absorption and longer serum half-life of 18 to 22 hours compared to other derivates of tetracycline. ${ }^{20,28}$

\section{CONCLUSION}

The results of this study indicate that the level of MMP is increased after provoked brain injury and can be inhibited by oral administration of doxycycline in rats. 


\section{DISCLOSURE STATETEMENT}

The author(s) declared no potential conflicts of interest with respect to the research, authorship, and/or publication of this article.

\section{FUNDING}

No funding was received for this study.

\section{REFERENCES}

1. Winkler EA. et al. Cerebral Edema in Traumatic brain injury. Neurosurgery Clinics of North America. Elsevier Inc 2016; 27(4): pp. 473-88. doi: 10.1016/j.nec.2016.05.008.

2. Zeng J. et al. Emodin attenuates brain edema after traumatic brain injuryin rats, 2017;10(11):15213-20.

3. Michinaga S. and Koyama Y. Pathogenesis of brain edema and investigation into anti- dema drugs, International Journal of Molecular Sciences 2015;16 (5): 9949-75. doi: 10.3390/ijms16059949.

4. Donkin JJ. and Vink R. Mechanisms of cerebral edema in traumatic brain injury: Therapeutic developments, Current Opinion in Neurology 2010; 23(3): 293-9. doi: 10.1097/ WCO.0b013e328337f451.

5. Weinstein E. Clinical review Doxycycline hyclate tablets. Center for drug evaluation and research. 2013: pp. 6.

6. Caprio RD, Lembo S, Costanzo LD, Balato A, Monfrecola G. Anti-inflammatory properties of low and high Doxycycline doses: An in vitro study. Hindawi Publishing Corporation 2015: 1-2.

7. Nascimento GC, Rizzi E, Gerlach RF, Leite-Panissi CR. Expression of MMP-2 and MMP-9 in the rat trigeminal ganglion during the development of temporomandibular joint inflammation. Braz J Med Biol Res 2013; 46: 956-67.

8. Shen LC, Chen YK, Lin LM, Shaw SY. Antiinvasion and antitumor growth effect of Doxycycline treatment for human oral squamouscell carcinoma-in vitro and in vivo studies. Oral Oncol 2010; 46: 178-84.

9. A. Marmarou, P.P. Fatouros, P. Barzo, G. Portella, M. Yoshihara, O. Tsuji, T. Yamamoto, F. Laine, S. Signoretti, J.D. Ward, M.R. Bullock, H.F. Young, Contribution of edema and cerebral blood volume to traumatic brain swelling in head-injured patients., J. Neurosurg. 93 (2000) 183-193

10. R.A. Nasution, A.A. Islam, M. Hatta, Prihantono, Decreased neutrophil levels in mice with traumatic brain injury after cape administration, Ann. Med. Surg. 54 (2020) 89-92.

11. Dixon CE, Clifton GL, Lighthall JW, Yaghmai AA, Hayes RL. A controlled cortical impact model of traumatic brain injury in the rat. J Neurosci Methods. 1991; 39:253-262. [PubMed: 1787745].

12. Hayashia T. Quantitative analyses of matrix metalloproteinase activity after traumatic brain injury in adult rats. Elsevier Brain research.2009:172-7.doi:10.1016/j. brainres. 2009.05.040

13. Webster KM. et al. Inflammation in epileptogenesis after traumatic brain injury. Journal of Neuroinflammation 2017; 14(1): pp. 1-17. doi: 10.1186/s12974-016-0786-1.

14. Wright G, Sharifi Y. Jover-Cobos, M. Jalan, R. The brain in acute on chronic liver failure. Metab. Brain Dis 2014; 4: 965-73.

15. Vajtr D. et al. Correlation of ultrastructural changes of endothelial cells and astrocytes occurring during blood brain barrier damage after TBI with biochemical markers of blood brain barrier leakage and inflammatory response. Physiological Research 2009; 58(2): pp. 263-8.

16. Yang Z, Fan R, Sun P, Cui H, Peng W. Rhubarb attenuates cerebral edema via inhibition of the extracellular signal-regulated kinase pathway following traumatic brain injury in rats. Phoog Mag 2018;14:134-9.

17. Robinson BD. Lomas A. Shaji CA. Isbell CL. Tharakan B. Doxycycline blocks matrixmetalloproteinase-9 and attenuates blood-brain barrier dysfunction and hyperpermeability after traumatic brain injury. The FASEB Journal 2017; 31(1).

18. Chen W. et all. Matrix metalloproteinases inhibition provides neuroprotection against hypoxia-ischemia in the developing brain. 2009. Journal of Neurochemistry. 111.726-36

19. Jung JJ. et all. Matrix metalloproteinase inhibitor, doxycycline and progression of calcific aortic valve disease in hyperlipidemic mice. Scientific Reports; 20166:32659. DOI: 10.1038/srep32659

20. Roeland RH, Lent NV, Sorsa T, Salo T, YrjöT 
Lindeman J. Inhibition of matrix metalloproteinases (MMPs) by tetracyclines. Tetracyclines in Biology, Chemistry and Medicine. 2001. London: Springer. pp 267-81.

21. Pasquale TR, Tan JS. Nonantimicrobial effects of antibacterial agents. Clin Infect Dis 2005; 40:12735.

22. Jin R, Yang G, Li G. Inflammatory mechanisms in ischemic stroke: role of inflammatory cells. J Leukoc Biol 2010. 87: 779-789,

23. Paulo W. et all. Doxycycline, a matrix metalloprotease inhibitor, reduces vascular remodeling and damage after cerebral ischemia in stroke-prone spontaneously hypertensive rats Am J Physiol Heart Circ Physiol. 2011. 301: 87-97

24. Alvarez JL, Krishnamurthy J, Teale JM.

Doxycycline treatment decreases morbidity and mortality of murine neurocysticercosis: evidence for reduction of apoptosis and matrix metalloproteinase activity. The American Journal of Pathology. 2009. 175(2):685-95

25. Burggraf D, Trinkl A, Dichgans M, Hamann GF. Doxycycline inhibits MMPs via modulation of plasminogen activators in focal cerebral ischemia. Neurobiol Dis. 2007; 25:506-13

26. Meli DN, Coimbra RS, Erhart DG, Loquet G, Bellac CL, Tauber MG, Neumann U, Leib SL. Doxycycline reduces mortality and injury to the brain and cochlea in experimental pneumococcal meningitis. Infect Immun. 2006; 74:3890-6.

27. Damian N. et al. Doxycycline reduces mortality and injury to the brain and cochlea in experimental pneumococcal meningitis. Infection and Immunity 2006; 74(7):3890-6.

28. Holmes NE, Charles PGP. Safety and efficacy review of Doxycycline. Clinical Medicine: Therapeutics 2009; 1: pp. 472-74. 